\title{
Meeting report: Fourth Summer School on Innovative Approaches for Identification of Antiviral Agents (IAAASS).
}

Stuart F.J. Le Grice ${ }^{a^{*}}$, Elias Maccionib ${ }^{b}$ Angela Corona ${ }^{b}$, Cristina Parolin ${ }^{d}$ and Enzo Tramontano ${ }^{b}$

${ }^{a}$ Basic Research Laboratory, National Cancer Institute, Frederick, MD, USA;

${ }^{b}$ Department of Life and Environmental Sciences, University of Cagliari, Cittadella Universitaria SS544, 090542 Monserrato, Italy.

${ }^{c}$ Department of Molecular Medicine, University of Padova, via Gabelli63, 35121 Padova, Italy.

${ }^{*}$ Corresponding author

aBasic Research Laboratory, National Cancer Institute, Frederick, MD, USA;

Tel +1-301-846-5256

e-mail legrices@mail.nih.gov 


\section{Abstract}

The $4^{\text {th }}$ Summer School on Innovative Approaches for the Identification of Antiviral Agents (IAAASS) was held at the Sardegna Ricerche Research Park in Santa Margherita di Pula, Sardinia, Italy from September 24 - 28, 2018. The Summer School assembled 21 internationally recognized experts and 46 graduate and postgraduate students, with the goal of discussing advances in antiviral drug discovery from the perspective of high-throughput screening, medicinal chemistry, computational chemistry, virology, molecular and structural biology. The meeting format involved three components: (a) morning sessions of plenary talks/overviews from invited speakers, (b) afternoon sessions of posters and short presentations from student participants, and (c) informal small-group discussions between students and participating faculty. Plenary talks also featured a roundtable discussion of the pros and cons of moving into an academic career versus employment in the pharmaceutical industry, featuring individuals with experience in one or both arenas. The success of the IAAASS has come from placing emphasis on informal interactions, through which speakers made themselves available

to students throughout the Summer School. This report provides a summary of scientific contributions presented by the lecturers, with enclosed a supplementary file containing the abstracts of selected oral presentations and poster presentations at the $4^{\text {th }}$ IAAASS.

Keywords: summer school; drug discovery; antiviral strategies; high throughput screening; RNA-directed therapeutics; computational modeling. 


\section{Introduction}

What started as an evening discussion over dinner in Cagliari, Italy, in 2012 was realized in the first Innovative Approaches for Identification of Antiviral Agents Summer School (IAAASS). After discussing the need to encourage students to become involved in the antiviral research process and to make them understand the different souls involved in the field, a Summer School targeting those at late stages in their academic career was proposed later in the same year, from September 30 to October 4, 2012. The mission of the IAASS has been, from its very beginning, to provide a platform for informal discussions and exchange of ideas between students and internationally-recognized experts in a range of disciplines that ultimately focus on identification of small molecule antagonists and their translation into clinically-useful antiviral drugs. The initial IAAASS format combined morning session of plenary talks/overviews from invited speakers, afternoon informal small-group discussions between students and participating faculty and evening session of posters and short presentations from student participants. All the participants had to contribute by presenting their work.

The school was meant to be an occasion of informal learning and mentoring, and, for this reason, one of the key points was to locate academic activities, meals and overnight stays in a common structure to encourage maximum interaction, not only for the time of classes/discussions but also during meals and after dinner. This simple organizational aspect, together with the generous availability of the teachers, allowed the establishment of an informal atmosphere between teachers and students and has certainly facilitated that meeting/confrontation between people experienced and less experienced in science, which was the purpose of the initiative. The first edition was a success, with the participation of 32 students from all over the world.

The success of this experimental venture was replicated every two years, with small improvements and adjustments every time. Since the $2^{\text {nd }}$ edition (September 28 - October 3 , 2014) a Round-table of discussion about working perspectives for post-docs was introduced. 
Form the $3^{\text {rd }}$ edition (September 28 - October 3, 2016), the school convened at the Science and Technology Park of Sardinia. Originating from the commitment of the Autonomous Regional Government of Sardinia and the European Union, the Science and Technology Park of Sardinia institution provides a system of advanced facilities and services fostering innovation, development and the translation of biomedical research. One of two centers is located in a strategic geographic area in the territory of Pula, in the hinterland of Cagliari, close to the Southwestern coast of Sardinia. Its grounds cover an area of 160 ha, set in a nature park at the foot of the Sulcis mountain range.

The $4^{\text {th }}$ IAAASS held at the Sardegna Ricerche Research Park from September $24-28$, 2018. The Summer School assembled 21 internationally-recognized experts and 46 graduate/postgraduate students, over $50 \%$ of whom were from international institutions outside Italy, with the goal of discussing advances in antiviral drug discovery from the perspective of high throughput screening, medicinal chemistry, computational chemistry, virology, molecular and structural biology. The school maintained the original format of plenary lectures, poster presentations, oral communications and discussion groups. In this edition, for the first time, the best oral communication and the best poster presentation were acknowledged with a small prize. Plenary talks also featured a roundtable discussion the pros and cons of moving into an academic career versus employment in the pharmaceutical industry, featuring individuals with experience in one, or both arenas. The success of this venture has come from placing emphasis on informal interactions, through which speakers made themselves available to students throughout the Summer School.

This report provides a summary of the scientific contributions presented by the lecturers and has a Supplementary File containing all the abstracts of student oral presentations (Appendix A), poster presentations (Appendix B), and recipients of travel awards (Appendix C).

\section{Overview}


Simplification of screening strategies (in some cases using everyday laboratory instrumentation), coupled with programs of synthetic chemistry, have brought drug discovery into the realm of many academic institutions. Such integration of drug discovery efforts, while not at a scale that matches that of the pharmaceutical industry, nevertheless gives students a first flavor of the multi-disciplinary effort necessary to advance the discovery pipeline from a biological target to a small molecule antagonist that may ultimately be considered for clinical evaluation.

An outstanding example of the drug discovery pipeline was provided by Professor Raffaele De Francesco, INMG, Italy, an integral contributor to an effort that has led to successful treatment of hepatitis $\mathrm{C}$ virus (HCV) infected individuals with direct acting antivirals (DAAs) to the extent that the percentage achieving a cure is nearing $100 \%$. This success is even more remarkable inasmuch as HCV was first identified in 1989. Figure 1 summarizes the remarkable timeline for development of HCV antivirals from 1989 to 2017.

In addition to plenary lectures, several mechanisms have been established to encourage/enhance student participation, including a poster session, selected oral presentations from students and an evening session of small group discussions. A routine challenge of the Summer School has been to encourage questions from students following either plenary talks or those of their peers, based almost exclusively on a perceived language barrier. Rising to this challenge, Professor Reuben Harris, University of Minnesota, introduced the highly successful "musical microphone" concept. In this strategy, rather than waiting for an individual to volunteer a question, microphones were handed to students prior to the presentation, giving them sufficient time to formulate their question. While somewhat daunting at the outset, this approach gradually found support in that student "volunteers" were then given the opportunity to pass the microphone to neighbor, thus motivating several colleagues to prepare a question. An indirect consequence of this strategy was that the question-and-answer component of all presentations was driven by students with input from plenary speakers rather 
than the converse. Achieving this informal and relaxed approach early in the Summer School was clearly beneficial for subsequent interactions.

\section{Inaugural Lecture}

Host-pathogen interactions and antiviral drug potency. José A. Esté, AIDS Research Institute-IrsiCaixa, Hospital Germans Trias i Pujol, Universitat Autónoma de Barcelona, Badalona, Spain.

Viruses are intracellular parasites requiring cellular machinery to replicate. Perturbations of the host cell metabolism by virus infections often lead to virus-induced cytophatic effects or aberrant cell proliferation. Additionally, virus infections trigger cell defense mechanisms aimed at inhibiting their replication, initiating and innate immune response and inflammation. Virus restriction factors are part of such response, comprising a number of intracellular proteins with antiviral properties that detect and recognize infections by identifying foreign proteins and/or genomic materials though pattern recognition receptors. Restriction factors may be associated with cell metabolic processes required for virus replication, including entry, genome processing, transcription and/or maturation. Viruses have developed mechanisms to counteract the antiviral effect of restriction factors. Professor Este' presentation reviewed cellular mechanisms controlling and regulating virus replication, using HIV-1 as a model, providing evidence to indicate how virus restriction factors affect virus replication and susceptibility to antimetabolites and antiviral drugs. The role of the HIV-1 restriction factor, SAMHD1, in controlling cell proliferation, the antiviral immune response and virus replication was reviewed, and how its activity may be modulated to combat virus infections.

Although antiretroviral therapy (ART) has significantly transformed virus infection from a terminal illness to a chronic manageable disease, HIV-1 persists in a latent state as an integrated provirus in resting memory CD4+ T cells, and no strategy to date has provided sustained control in the absence of ART. The latent reservoir is established very early during infection with a half-life of $\sim 44$ months. The latent reservoir is the major barrier to curing HIV-1 infection. To establish a productive infection, HIV-1 must counteract cellular innate immune mechanisms and redirect cellular processes toward viral replication. Recent studies have shown that HIV-1 and other primate immunodeficiency viruses subvert cell cycle regulatory mechanisms to achieve these ends. For example, the viral Vpr and Vpx proteins target cell cycle controls to counter innate immunity. Nevertheless, the innate immune response is an 
effective and advanced system that constitutes one of the first lines of defense against pathogenic infection. Detection of viruses by innate immune sensors induces protective antiviral immunity. Upon viral detection, activation of this host defense-mediated mechanism induces a family of protective cytokines, known as type I interferons (IFN). IFN signaling pathways regulate hundreds of genes involved in anti-viral, pro-inflammatory and anti-proliferative activity, promoting their use in treating several diseases, including viral infections, cancer and autoimmunity. During HIV-1 infection, viral pathogen-associated molecular patterns are recognized by pathogen-pattern recognition receptors (PPRRs) on infected cells, which trigger a signaling cascade that initiates innate intracellular antiviral defenses aimed at restricting the replication and spread of the virus. This cell-intrinsic response propagates outward via the action of secreted factors such as cytokines and chemokines that activate other immune cells and attract them to the site of infection and to local lymphatic tissue. Antiviral innate effector cells can subsequently contribute to control of viremia and modulate the quality of the adaptive immune response to HIV-1. The concerted actions of PPRR signaling, specific viral-restriction factors, innate immune cells, innate-adaptive immune crosstalk and viral evasion strategies determine, in part, the outcome of HIV-1 infection.

Single-stranded DNA (ssDNA) derived from HIV-1 activates the type I IFN-inducing DNA sensors cGAS-STING in a sequence-dependent manner. However, synthesis of HIV DNA during infection may be insufficient for immune activation and there are still discrepant results on the capacity of HIV-1 to activate innate immunity in immune cells such as macrophages. The Esté group and others have shown that established HIV-1 infection of monocyte-derived cells induces upregulation of the RNA PPRRs melanoma differentiation-associated protein 5 (MDA5) and retinoic acid-inducible gene I (RIG-I), production of IFN, and transcription of interferon stimulated genes (ISG). Pharmacological stimulation of the RIG-I pathway has been proposed as an alternative mechanism to kill cells in the latent HIV reservoir, following viral reactivation. Enhancement of RIG-I signaling ex vivo, was shown to increase HIV-1 transcription, and induce preferential apoptosis of HIV-infected cells, recapitulating the effect observed by chronic infection of macrophages.

SAMHD1 (sterile alpha motif and histidine-aspartate-domain-containing protein 1) is a member of a unique group of host restriction factors that limit retroviral replication at distinct stages of the viral life cycle. HIV-1 is relatively inefficient in infecting non-dividing cells, such as monocytes, dendritic cells (DCs), and quiescent CD4+ T cells. Infection of these cells can be 
enhanced by the HIV-2 accessory protein Vpx, based on the observed correlation between the ability of Vpx to associate with a ubiquitin E3 ligase complex to induce SAMHD1 degradation and enhance reverse transcription. SAMHD1 is a triphosphohydrolase enzyme that controls the intracellular level of deoxyribonucleoside triphosphates (dNTPs) and this mechanism explains, in part, the role of SAMHD1 as an HIV restriction factor. However, recent findings have led researchers to question the depletion of the intracellular dNTP pool as fully determining SAMHD1 function, adding complexity to the mode of action and role of SAMHD1 in cell proliferation, immune control and virus replication. A previously-recognized but challenged exonuclease activity of SAMHD1 has been recently revamped by the finding that SAMHD1 promotes degradation of nascent DNA by stimulating an exonuclease activity that forms part of the MRN (Mre11-Rad50-Nbs1 complex) which plays a central role in double-strand break (DSB) repair, DNA recombination, maintenance of telomere integrity and meiosis. The function of the MRN complex has been associated to innate immune signaling.

The Esté group has extensively studied the role of SAMHD1 in disease and in particular its effect in regulating HIV-1 replication, cell cycle control and innate immune function, showing that it modifies the efficacy of a wide variety of agents used to treat infections, cancer and other diseases. Modulation of SAMHD1 function has the potential to improve antimetabolite-based antiviral and anti-cancer therapies, paving the way to the use of treatment combinations that fine tune antiviral and anti-cancer drug efficacy. His group has identified drugs and drug combinations that modulate SAMHD1 function, providing important tools to decipher and understand it role in innate immunity and disease. As their ability to effectively up- or downmodulate SAMHD1 function with validated pharmacological agents, a long-term goal is to evaluate the clinical use of SAMHD1 targeting agents.

Mounting evidence suggests that HIV-1 persistence is the product of clonal expansion of latently infected cells and that HIV integration into genes associated with cancer or cell-cycle regulation appears to confer an advantage that allows these cells to survive therapies that target HIV-infected proliferating cells may contribute to a curative strategy for HIV infection. The Esté group supports SAMHD1 as a target through which control of virus replication, eradication of virus-infected proliferating cells, and regulation of the innate immune system may be achieved, providing a new avenue towards improved treatment and cure of HIV+ individuals.

\section{Plenary Talks}




\section{Day 1:}

Structural targeting signal transduction pathways to fight the flu. S. Ludwig, Institute of Virology (IVM), University of Muenster, Germany.

Summary: Infection by RNA viruses, such as influenza viruses (IAV) results in the activation of a variety of intracellular signaling responses. While most of these pathways are part of a cellular defense strategy to fight the invading pathogen, IAV exploit some of these activities to support efficient replication. This dependence of IAV on cellular signaling factors provides opportunities for a novel antiviral strategy that targets essential host factors instead of viral components. Professor Ludwig's group identified the classical mitogenic MAP kinase cascade as a suitable axis for antiviral intervention, demonstrating that inhibition of this pathway efficiently blocked virus replication in cells and animals. MEK inhibitors are now under advanced clinical evaluation or even licensed for clinical use for other diseases. He further demonstrated that these novel signaling blockers (a) efficiently inhibit influenza virus replication in vitro and in vivo, (b) are broadly active against all influenza A and B viruses analysed so far, (c) are active against oseltamivir resistant viruses, (d) are not toxic for cells or animals in the concentration and time line used, (e) display an enhanced treatment time window compared to standard of care, and (e) confirm the postulated mode of action: blockade of the export of viral genomes from the nucleus. Professor Ludwig's presentation finished by describing the latest developments in the strategy of repurposing MEK inhibitors.

Small molecule targeting of viral, virus-coded and cellular IncRNAs. S. Le Grice, National Cancer Institute, Frederick, MD, USA.

Summary: Traditional therapeutic strategies have focused on targeting enzymes and structural proteins that are critical to virus replication. However, an increasing body of evidence in the literature describes small molecules that target structured motifs of both viral RNA genomes and virus-coded RNAs (e.g. pseudoknots, bulges and hairpins) which are often highly conserved 
and critical to RNA function. Several recent examples have been cited where in vitro and in silico high throughput screening (HTS) have uncovered small molecules that selectively bind RNA motifs, with corresponding physiological effects in cell culture or animal models. In their favor, the pharmacokinetics of small molecules are generally superior to those of oligonucleotides, whose anionic character limits cell permeability and systemic distribution. Dr. Le Grice's presentation summarized ongoing efforts at the National Cancer Institute to develop and improve HTS strategies. Viral and cellular RNAs under investigation included pregenomic RNA (pgRNA) of hepatitis B virus (HBV) and genomic RNA of Dengue virus (DENV), Kaposi's sarcoma herpesvirus (KSHV) PAN IncRNA and the cellular IncRNA MALAT1. Finally, he described a simplified HTS approach that would find use in most academic laboratories.

Computer-aided design of novel antivirals. A. Brancale, Cardiff University, UK.

Summary: Drug discovery is a high risk, high gains enterprise. Computer modeling techniques help lower this risk element significantly and are now an integral part of the modern drug discovery process. These techniques allow faster hit identification and lead optimization phases of drug development by evaluating novel potential drugs in silico before committing to their chemical synthesis. This not only offers an enormous advantage in time but also on costs, as synthesis of novel compounds is often the slow and expensive step at the outset of a drug discovery project. Millions of candidates can often be screened for the cost of one multi-step synthesis. In addition to these applications, molecular modeling techniques are becoming increasingly useful in understanding more complex biological processes. For example, by examining how proteins interact with each other within a specific biological pathway, it is possible to identify specific mechanisms. As target for novel therapeutics. In his presentation, Professor Brancale provided examples of the molecular modelling techniques that have successfully been applied in antiviral drug design, highlighting the strengths and limitations of these approaches. 
Functionality and druggability of the E6 oncoprotein of HPV. G. Palù, University of Padova, Italy.

Summary: High-risk human papillomaviruses (HR-HPVs) are the causative agents for the onset of several epithelial cancers in humans. Deregulated expression of the viral oncoproteins E6 and E7 is the driving force sustaining the progression of malignant transformation. Targeting the viral oncoproteins through inhibitory compounds represent an intriguing strategy to treat HPVassociated neoplasias. Professor Palu reported his group's investigation on the homodimerizarion process of E6 and on its binding to p53, proposing a model in which E6 binds p53 as a monomer in the nucleus while forming a dimer in the cytoplasm where it interacts with other cellular partners through the PDZ domain. This led to the postulation that the dimeric form of E6 can play a relevant oncogenic role in allowing the nuclear accumulation of TAZ, a master transcription regulator in cancer stem cells, normally retained at the membrane-cytoplasmic level via protein-protein interactions (PPI) with Scribble. Professor Palu's group also confirmed the structural data that the E6 homodimerization interface overlaps with the E6/p53 binding interface and identified, by in silico analysis, a crucial cavity as a molecular target for therapeutic intervention. An optimized cell-based assay for screening putative anti-E6 compounds blocking the degradation of p53 in living cells was developed. This strategy identified a lead compound that antagonized E6-mediated degradation of p53, restored p53 levels and activity, and induced senescence of HPV-positive cells. These findings represent an important first step towards the successful development of anti-HPV drugs.

\section{Day 2:}

From the elucidation of HCV life-cycle to the development of highly effective antivirals. $R$. De Francesco, INMG, Italy. 
Summary: Prof. De Francesco provided a remarkably short timeline for HCV antiviral drug discovery and development. Since the discovery of the causative agent of non-A non-B hepatitis in 1989, treatment of chronic hepatitis C virus (HCV) with antivirals has made astonishing progress, culminating in the development of interferon-free, all-oral combinations of direct-acting antiviral agents (DAAs) that can cure HCV infection in $>95 \%$ of patients, including populations for whom resolution of the infection has been traditionally difficult to achieve. Various DAA classes now available that target the HCV NS3/4A protease, the NS5A protein or the NS5B polymerase. The last of these viral enzyme offers several target sites: the catalytic domain for nucleoside/nucleotide analogues and different allosteric sites for non-nucleoside inhibitors. Although some medical challenges remain, there are multiple available combination regimens, including with and without ribavirin, and the decision of what treatment to prescribe to what patient requires knowledge of viral genotype, prior treatment failures, pre-existence of drugresistant viral variants, as well as an assessment of liver disease stage. New fixed-dose combination regimens that are truly pan-genotypic, ribavirin-free, and insensitive to the presence of viral variants resistant to previous antivirals, have been now approved, holding the promise to further remove complexity and add effectiveness to the treatment for chronic hepatitis $\mathrm{C}$.

Discovery of Grazoprevir an HCV pangenotype protease inhibitor approved for interferon free therapy to cure HCV infected patients. Vincenzo Summa, IRBM Science Park, Pomezia, Italy.

Summary: Professor Summa provided a historical perspective on development of MK-5172, known as Grazoprevir, a second-generation HCV NS3/4a protease inhibitor. Its discovery was a journey through all aspects of the drug discovery process, and he highlighted the strategies applied to overcome issues encountered during this very challenging and competitive project. HCV NS3/4a protease is a proven therapeutic target for the treatment of chronic hepatitis C 
virus infection. The presentation described the discovery and evolution of the first peptide product inhibitor with micromolar activity to a series of peptidomimetics. These achieved subnanomolar or nanomolar activity in enzyme or cell based assay for genotype $1 \mathrm{a}, 1 \mathrm{~b}, 2 \mathrm{a}, 2 \mathrm{~b}$ and 3a proteases and against a large panel of resistant mutations identified either during clinical evaluation of HCV protease inhibitors or by similar selection in the replicon cell system, in particular against the clinical relevant mutations $1 \mathrm{~b}$ R155K and D168Y. Potent activity in the low nanomolar range was also observed in a cell-based HCV replicon assay both in the presence of 10\% FBS (genotypes 1b and 2a) and 50\% NHS (genotype 1b). MK-5172 retains subnanomolar potency against a panel of $1 \mathrm{a}$ and $1 \mathrm{~b}$ sequences isolated from naïve patients suggesting the agent will be less sensitive to the natural genetic diversity. Based on favorable pangenotype activity and pharmacokinetic profiles, MK-5172 (Grazoprevir) was selected for clinical development as a second-generation HCV protease compound in combination with Elbasvir. This combination, known as Zepatier, was successfully approved by FDA and EMEA for peg interferon free therapy in 2016. The teams that discovered Grazoprevir and Elbasvir were appointed as Heroes of Chemistry in 2017 by the American Chemical Society. The discovery of Grazoprevir ${ }^{\mathrm{TM}}$ is a very good example of interdisciplinary team work and creative medicinal chemistry strategy.

\section{Day 3:}

Progress in development of inhibitors of viral reverse transcriptase. Z. Janeba, Institute of Organic Chemistry and Biochemistry, Czech Academy of Sciences, Czech Republic.

Summary: Professor Janeba provided an update on the latest development of antiviral agents targeting HIV reverse transcriptase (HIV RT) Three therapeutic classes of RT inhibitors are routinely used in the clinic to treat HIV-1 infection: nucleoside reverse transcriptase inhibitors (NRTIs), nucleoțide reverse transcriptase inhibitors (NtRTIs), and non-nucleoside reverse transcriptase inhibitors (NNRTIs). NRTls (e.g. zidovudine) are usually analogues of 
deoxynucleosides that lack a 3'-OH group on the (pseudo)sugar moiety. To exert antiviral activity, NRTIs must be first converted by host-cell kinases to the corresponding triphosphate analogues. NtRTIs (e.g. tenofovir) are nucleoside phosphonates and, unlike the NRTIs, require only double phosphorylation (to phosphonate-diphosphates). Both classes of compounds then act as DNA chain terminators. NNRTIs (e.g. efavirenz and rilpivirine) are structurally distinct from nucleoside analogues. They are small hydrophobic molecules and do not require intracellular activation. Unlike NRTIs and NtRTIs (binding to the active site of HIV RT), the NNRTIs bind to the so-called NNRTI binding pocket that is situated about $10 \AA$ away from the RT active site.

\section{Antisense oligonucleotides for the control of gene expression in disease and infection.}

M. L. Hastings. The Center for Genetic Diseases, Chicago Medical School, USA.

Summary: Antisense oligonucleotides (ASOs) are synthetic molecules comprising nucleotides or nucleotide analogues that bind to a sequence by complementary base-pairing. The specific base-pairing of these short, antisense nucleic acids to the targeted RNA results in degradation or modification of the RNA, and in this way, ASOs can control gene expression. ASOs have achieved clinical success in the treatment of human diseases and pathological conditions. Given this clinical success, ASOs becoming widely regarded as a powerful therapeutic platform. Professor Hastings reviewed efforts of her group using antisense technology to develop therapeutics for a number of neurodegenerative and neurosensory disorders such as Usher syndrome, Batten disease and Alzheimer's disease. ASOs are also being used in anti-viral approaches to directly down-regulate viral RNA or to control expression of endogenous genes required for viral infection. The use of antisense technology for the treatment of disease and their emerging application as anti-viral agents was also discussed. 
Targeting evolvability. R. S. Harris, Howard Hughes Medical Institute, University of Minnesota, USA.

Summary: Cancers often display incredible genetic heterogeneity characterized by hundreds of gross chromosomal aberrations and tens of thousands of somatic mutations. Tumor evolution is thought to be ongoing and the sum of all exogenous and endogenous mutagenic processes. Many viruses evolve at much higher rates, and virus evolution is thought to be similarly complex, with viral and cellular processes contributing to the observed high rates of mutation. Historically, tumor and virus evolution have been thought of as largely independent processes. However, many studies have converged recently on the antiviral APOBEC3 enzymes as a major source of virus and tumor mutagenesis and evolution. Professor Harris' lecture highlighted recent progress in these areas, specifically data showing how papillomaviruses (HPV) and polyomaviruses (JCV/BKV) specifically upregulate APOBEC3B, and results demonstrating a newly discovered mechanism of APOBEC3B inhibition by the $\gamma$-herpesviruses EBV and KSHV. Finally, Professor Harris' lecture discussed pros and cons of therapeutically targeting evolvability as a potentially transformative anticancer and antiviral strategy.

Antiviral compounds with broad spectrum activity on enveloped viruses. M. Botta Dipartimento Biotecnologie, Chimica e Farmacia, Università degli Studi di Siena, Italy.

Summary: Viral infections are one of the ten leading causes of death worldwide. Nowadays, although effective antiviral strategies have been successfully developed for some important pathogens such as HIV and HCV, antiviral drugs or vaccines are still missing for the majority of viruses. As an example, no effective antiviral strategies are yet available for viruses causing chronic infections such as HBV (1), as well as for tropical viruses like Dengue virus and Zika (ZIKV). In this scenario, the possibility to find antiviral molecules directed against viral envelopes is of particular interest in order to identify broad spectrum antiviral drugs. The selectivity of these strategies is based on the fact that viral envelopes are static and characterized by an absence 
of repair mechanisms, in contrast with the biogenic membranes of the cells that are endowed with plenty of tools to repair membrane damage or alteration. Professor Botta highlighted a series of potent molecules developed in his group that displayed nanomolar activities against HIV-1. These compounds also displayed significant potency against Herpes simplex viruses 1 and 2, but were completely ineffective against HPV, a non-enveloped virus. His results suggested that the mode of action of molecules developed in his lab could involve the viral envelope. His group subsequently investigated activity of these compounds against an acyclovir-resistant strain of HSV-2 and 6 other important enveloped pathogens such as ZIKV, Influenza virus and Respiratory Syncytial virus, and confirmed its complete inactivity against three non-enveloped viruses. Mechanism of action studies identified a lipid oxidizing activity and the impairment of viral entry of HSV-2.

\section{Day 4:}

Re-emergence of antibodies in antiviral therapy. T. Mertens, Institute of Virology Ulm University Hospital, Germany.

Summary: Professor Mertens highlighted that polyclonal or monoclonal antibody preparations have been licensed to treat certain infections (e.g. postexposure prophylaxis for Hepatitis-BVirus, varicella-zoster virus or rabies virus, preexposure prophylaxis for at-risk persons (including pregnant women) for Cytomegalovirus or infants with pulmonary pathologies to prevent Respiratory syncytial virus). In the 20th century striking advances were made in the research of active vaccines, antibiotics, antivirals, and T-cell immunology. A range of human polyclonal Ig products has been available for more than 20 years, but antibodies were no longer a main research focus. New possibilities of molecular characterization of relevant antigens and antibody specificities (e.g. super neutralizing antibodies) together with hybridoma technology and new molecular recombinant technologies has revived research on antibodies not only for prevention and treatment of infectious but also for treatment of cancer and immunomodulation. 
There is renewed activity e.g. in HIV where first-generation monoclonal antibody combinations were found to be largely ineffective but new-generation antibodies derived using single-cellbased antibody cloning methods have revealed more potent broadly neutralizing activity against HIV-1. These include also MAbs to host cellular receptors. In some cases, a mixture of MAbs may be required to provide multiple epitope recognition like polyclonal sera. Many developments in rDNA technology in the 1980s and 1990s have enabled the expression of high levels of recombinant MAbs in defined CCLs, the chimerization or humanization of mMAbs, the isolation of human MAbs (hMAbs) from transgenic mice, and the screening for specific hMAbs from a "library" of MAbs. At present the number of new antibodies for therapy is exponentially increasing.

Disrupting HIV nucleocapsid protein with mercaptobenzamides. D. H. Appella, NIDDK, $N I H$, USA

Summary: Dr. Appella's laboratory has been involved in the study and development of mercaptobenzamide-derived molecules that inhibit HIV maturation via a unique mechanism of action. These molecules unfold the HIV Gag polyprotein by acetylating highly conserved cysteine and lysine residues within the matrix, capsid, nucleocapsid (NC), and p6 domains of Gag. Within cells, mercaptobenzamides react intracellularly with acetyl CoA to form an S-acetyl thioester intermediate. This S-acyl-mercaptobenzamide intermediate reacts directly with free cysteine thiols in Gag to form S-acetyl cysteine that can subsequently transfer the acetyl group to the amines of nearby lysine residues. This acetylation process first unfolds NC by promoting irreversible ejection of zinc from the zinc fingers. Then, unfolding propagates to the other domains of Gag. Upon reaction with Gag, the original mercaptobenzamide molecule is regenerated and then re-enters the acetyl CoA acylation pathway. His work suggests that the mercaptobenzamide acts as a catalytic HIV inactivator where a single molecule has the potential to inhibit multiple copies of Gag. Treatment of HIV-infected cells with the 
mercaptobenzamide or prodrug derivatives results in crosslinking and mis-processing of the Gag polyprotein, leading to production of non-infectious virus particles. His group has been actively examining the antiviral activity, formulation, and translation of prodrug derivatives as a microbicide for protection against HIV infection or as an oral treatment for HIV infection.

Viral DNA polymerases: effects of amino acid substitutions on viral adaptation and evolution. G. Andrei, KU Leuven, Belgium

Summary: Most virologists believe RNA viruses are inherently variable in contrast to stable DNA viruses due to the lack of proofreading activity by most RNA-dependent polymerases. However, recent data suggest that diversity and evolution of herpesviruses are impacted by different mechanisms other than the usual consideration of DNA polymerase fidelity. Professor Andrei's laboratory investigates a novel mechanism that may contribute to herpesvirus and poxvirus genetic diversity, namely mutations in DNA polymerases affecting the fidelity of DNA replication. Substitutions in the $3^{\prime} \rightarrow 5^{\prime}$ exonuclease region of DNA polymerases may result in decreased proofreading capacity, leading to the appearance of spontaneous mutations. A substitution in the DNA polymerase that induce a high frequency of mutations in the genome is known as a mutator phenotype. Specific changes in the $3^{\prime} \rightarrow 5^{\prime}$ exonuclease region of the human polymerases $\beta$ and $\delta$ revealed that these variants had a mutator phenotype contributing to the etiology of some cancers by inducing mutations and genomic instability. Among viruses, a DNA polymerase mutator phenotype has been described for bacteriophage T4 and in poxviruses by her team. Her group also identified a mutator phenotype in herpesviruses, analyzing its replication capacity in competition with wild-type virus in the presence or absence of drugs and following the evolution of viral populations by next generation sequencing. Her group is are currently using this novel approach to determine the dynamics of herpesvirus populations, in contrast to studies that evaluate replication capacity in mono- infections and without drugpressure. 
In silico virtual screening approaches for antiviral drug discovery. S. Distinto, Computer Aided Drug Design Lab - Department of Life and Environmental Sciences, University of Cagliari, Italy.

Summary: Dr. Distino provided an overview of methods most commonly employed for virtual screening (VS) against viral targets Medicinal chemistry has become increasingly dynamic and medicinal chemists face the challenge of rapidly evolving new technologies. In the last decade, medicinal chemistry methodologies have been largely replaced from an individual scheme to an interdisciplinary approach. Furthermore, the shift from traditional to Omics-based applications needed to develop computational, chemo and bioinformatic tools that could help medicinal chemists to analyze, link, and compare the research results. Hence, inevitably, drug research has necessarily oriented drug discovery toward more rational strategies. In silico VS is one of the most promising approaches to accelerate the drug development process. An efficient analysis of key compound and target properties (e.g. structural, physicochemical, biochemical, pharmacological, toxicological) is crucial for carrying out a virtual screening process. At the same time, it can reduce the attrition rates in drug development. The main purpose of VS is to identify novel chemical scaffolds as hits for further optimization by using medicinal chemistry approaches.

\section{Round-Table Discussion: Choosing an Academic vs Pharmaceutical Career}

Panelists: Dr. Stuart Le Grice, National Cancer Institute, USA; Dr. Vincenzo Summa, IRBM, Italy; Dr.Timothy Tellinghuisen, Hoffmann-La Roche, Switzerland, Dr. Franco Lori, Virostatics, Italy.

A recurring event of the Summer School was a 90-minute round-table discussion with student participants and a group of four panelists on the transition from their academic studies to an 
academic career or a career in the pharmaceutical industry. Bringing 25 years of extensive experience in Biotech management, Dr. Lori has overseen the successful start-up of three biotechnology companies. After 7 years with Hoffman-La Roche, Switzerland, Dr. Le Grice pursued a 10-year academic career in the United States, finally being recruited to the National Cancer Institute - Frederick in 1989. In contrast Dr. Tellinghuisen's career spanned academic studies at the University of Massachusetts, Purdue University, The Rockefeller University, The Scripps Research Institute, and ultimately to Hoffmann-La Roche as the Head of Virology and Discovery Area Leader. Finally, after 13 years with Merck, Dr. Summa moved to IRBM Science Park, a Research center formally a spin-off of the Merck Research Laboratories, Rome, where he is currently Executive Director of Chemistry and Vice President of Drug Discovery. Panelist shared their views and decisions for their ultimate career choice, with the ensuing discussion answering questions from the audience. Since all panelists shared a common theme of working in the pharmaceutical industry, efforts were made to demonstrate the value of team and goaloriented research as opposed to academic research, which often suffers from working in isolation ("silos"). As a consequence, projects that are "green-lighted" in the pharmaceutical industry proceed at a speed that can rarely be matched by academic researchers. At the same time, one consequence of goal-oriented pharmaceutical research is the unexpected termination of projects based on factors that might be unrelated to their quality. In this respect, panelists agreed that close involvement with a project should be combined with a sense of detachment and willingness to move on to new priorities as the remand requires. For students who prefer to follow an academic career, it was important to point out to students that successful biomedical research requires a multi-disciplinary approach combining biological, biochemical, chemical and structural expertise, much that same as in the pharmaceutical industry. Finally, as the final career decision is a personal one, students were encouraged to explore the possibility of performing post-doctoral research in the pharmaceutical industry to experience the concept of team-oriented research. 


\section{Small-group discussions}

At the conclusion of previous IAAASS meetings, students were invited to respond to a survey on the merits and drawbacks of the Summer School, prominent among which was the success of informal small-group discussion teams. These 90-minute meetings were conducted in the evening at the Hotel Flamingo residence following student presentations and involved a threeday rotation system comprising 6-8 students and two plenary speakers. No strict agenda was followed, with discussion themes varying from development of high throughput screening strategies, de novo drug design via computational chemistry and medicinal chemistry, to the role of women in science. Although the funding mechanisms varied by country, advice was sought by those seeking an academic career on grant-writing skills. Most importantly, this mechanism allowed students a means direct and informal contact with leaders in their respective fields in a manner that might not be possible at larger scientific meetings. Given the international composition of the Summer School, group discussions were initiated by speakers giving a synopsis of their scientific background, followed by each student participant providing a short summary of her/his current research. The goal of this approach was to stimulate interaction between students to the extent that, towards the end of the Summer School, group leaders were active participants in discussions led by students.

A discussion theme of particular interest was the future of women in science and opportunities provided by academic institutions to help them pursue their career. Several examples were cited where female participation in the biological sciences at the graduate and post-graduate level was high, only to rapidly decline with respect to obtaining an early career faculty position. Initiatives to encourage women at early stages of their career, where in many instances the demands of combining a competitive laboratory with those of raising a family must be balanced, were discussed. 


\section{Poster and oral presentation prize-winners}

An additional feature of the $4^{\text {th }}$ IAAASS was three awards recognizing the most meritorious poster and oral presentations, as judged by members of the Organizing Committee and select plenary speakers. Presentations were judged on three criteria, namely (a) the visual quality of the presentation, (b) the oral presentation and (c), the response of the speaker to questions of the audience or judging panel. Two oral presentations were selected for recognition, both addressing herpes simplex virus (HSV) infection. Ms. Luisa Fernanda Duarte, Pontificia Universidad Catolica de Chile, presented data describing the "Antiviral effects of Cetylpyridinium chloride (CPC) over the replication cycle of Herpes Simplex Viruses". CPC is a cationic quaternary ammonium compound that is added to mouthwashes and toothpastes as an antiseptic with a broad antimicrobial spectrum. While bactericidal and fungicidal effects have been widely reported for CPC, little research has been invested in its antiviral properties In this presentation, human gingival fibroblasts and epithelial cells treated with CPC displayed reduced infection with HSV strains that encode structural and non-structural versions of the green fluorescent protein (GFP). These results suggest that CPC as an inhibitor of HSV replication and that the blockade occurs after virus entry and prior to genome replication takes place. Future use of CPC in topical formulations might therefore be considered for HSV infections.

A second award was given in recognition of the oral presentation "Characterization of the Humoral Response Directed against Herpes Simplex Virus Glycoproteins in Naturally-infected patients" by Ms. Elena Criscuolo, "Vita Salute" San Rafael University, Milan. Current HSV antiviral strategies are burdened by the emergence of drug resistance and toxicity. In the absence of a vaccine, novel alternative anti-HSV compounds are thus needed. This group has identified a human monoclonal antibody targeting HSV glycoprotein D $(g D)$ that inhibited replication of wild type and drug resistant HSV clinical isolates in vitro both prior to and following virus entry. Viral $\mathrm{gD}$ is one of the most immunogenic HSV proteins able of eliciting both neutralizing and non-neutralizing Abs in vivo. In vivo studies subsequently demonstrated that a 
single intravenous administration performed after HSV-1 and HSV-2 ocular and vaginal lethal challenge fully protected mice from severe disease and death.

Finally, a single award was made for the poster presentation of Mr. Harvijay Singh, Indian Institute of Technology Roorkee, Uttarakhand, entitled "Structure-Based Identification of Peptidometic Inhibitors of Chikungunya Virus and Determination of their Antiviral Activity" Chikungunya Virus (CHIKV) is an arbovirus responsible for causing fever and arthritis in humans, and against which no antivirals or vaccine is available. CHIKV protease (nsP2pro), responsible for maturation of the viral nonstructural polyprotein to form the replication complex, represents an important antiviral target. Based on the nsP2pro crystal structure, an octapeptide corresponding to the nsP3/4 cleavage site was docked into the active site. This initial study led to development of several peptidometic inhibitors. Two of these, designated Pep-I and Pep-II, inhibited nsP2pro activity in vitro, with $\mathrm{IC}_{50}$ values of $34 \mu \mathrm{m}$ and $42 \mu \mathrm{M}$, respectively. Pep-I and Pep-II were further evaluated for antiviral activity and demonstrated to inhibit CHIKV replication in BHK-21 cells. Identification of such peptidometic compounds opens new avenues for antiviral strategies against chikungunya infection. Student oral presentations, poster presentations, and recipients of travel awards are summarized in the Supplemental File.

\section{Acknowledgements}

The IAAASS Organizing Committee comprises Dr. Stuart F.J. Le Grice form National Cancer Institute-Frederick, USA, Professors Enzo Tramontano and Elias Maccioni, University of Cagliari, Italy, Professor Cristina Parolin, University of Padova and Dr. Angela Corona, University of Cagliari, Italy, with administrative support from Dr. Nicole Grandi and from Co.S.Me.Se, a university consortium promoted by the Universities "Magna Græcia" of Catanzaro, Cagliari and "Amedeo Avogadro" of Novara that has among its aims the doctoral and post-graduate training through the activation of initiatives aimed at expanding the scientific knowledge of the young graduates in the Pharmacological field. 
The $4^{\text {th }}$ IAAASS had the patronage of the University of Cagliari, the Società Italiana di Microbiologia Generale e Biotecnologie Microbiche and the Società Italiana di Virologia - Italian society for Virology. Financial support from Sardegna Ricerche, Roche Pharmaceuticals and Gilead is gratefully acknowledged, while the European Society for Virology, the Federation of European Microbiological Societies, the International Antiviral Symposium Foundation and Fondazione di Sardegna provided travel grants and support for student accommodation.

\section{Figure legend}

Figure 1. Timeline of HCV drug development. Only select accomplishments have been presented, and a more thorough list is available at https://www.hepatitiscentral.com/medications-to-treathepatitis-c-a-timeline/ 